\title{
Schwere bullöse arzneimittelinduzierte Hautreaktionen
}

\section{Severe Bullous Drug-Induced Skin Reactions}

M. Mockenhaupt, Y. Liß

Dokumentationszentrum schwerer Hautreaktionen (dZh), Universitäts-Hautklinik Freiburg

\section{Lernziel \\ $\nabla$}

Unter der Vielzahl von arzneimittelinduzierten Hautreaktionen finden sich verschiedene bullöse Reaktionsformen, deren klinische und ätiologische Einordnung selbst für erfahrene Dermatologen oft schwierig ist. Das frühzeitige Erkennen der schweren Reaktionsformen und ihre Behandlung stellen eine große Herausforderung dar. Im Folgenden sollen klinisches Bild, diagnostische Verfahren, differenzialdiagnostische Einordnung, aber auch auslösende Faktoren, pathogenetische Überlegungen und therapeutische Optionen dargestellt werden.

\section{Einleitung}

Schwere bullöse Arzneimittelreaktionen der Haut schließen das Stevens-Johnson-Syndrom (SJS), die toxisch epidermale Nekrolyse (TEN) wie auch deren Übergangsform und das generalisierte bullöse fixe Arzneiexanthem (generalized bullous fixed drug eruption; GBFDE) ein. Insgesamt sind diese schweren arzneimittelinduzierten Hautreaktionen selten, wobei nur für SJS und TEN verlässliche Inzidenzzahlen vorliegen, die zwischen 1,5 und 1,8 pro eine Mio. Personen pro Jahr schwanken [1]. Die genannten Reaktionsformen unterscheiden sich nicht nur in ihrem klinischen Bild, sondern auch hinsichtlich der auslösenden Arzneimittel, ihrer Prognose und Behandlung. Da es sich meist um arzneimittelinduzierte Reaktionen handelt, muss zudem das auslösende Agens rasch ermittelt und abgesetzt werden. Dann kommen therapeutische Interventionen zum Tragen, die je nach Reaktionsform supportive und symptomatische Maßnahmen oder systemische immunmodulierende Therapien beinhalten.

\section{Klinisches Bild und diagnostische Kriterien \\ $\nabla$}

\section{Stevens-Johnson-Syndrom und toxisch epidermale Nekrolyse}

Das Stevens-Johnson-Syndrom (SJS) wurde erstmals von den gleichnamigen amerikanischen Kinderärzten 1922 beschrieben. Die toxisch epidermale Nekrolyse (TEN) wurde vom schottischen Dermatologen A. Lyell 1956 publiziert und später häufig als Lyell-Syndrom bezeichnet. Beide Reaktionen weisen Erytheme mit Übergang in eine zum Teil ausgedehnte Blasenbildung auf, ähnlich einer großflächigen zweitgradigen Verbrennung. Gleichzeitig liegen erosive Schleimhautveränderungen vor, die in erster Linie die Mundhöhle, die Lippen, die Konjunktiven und das Genitale betreffen, aber auch Nasen-, Analoder Bronchialschleimhaut einschließen können (๑ Abb. 1, $\odot$ Abb. 2).

SJS und TEN gehen mit Fieber und oft ausgeprägtem Krankheitsgefühl einher [1]. Für Jahrzehnte waren die klinische Einteilung sowie die Nomenklatur der schweren Hautreaktionen sehr uneinheitlich. Dabei wurden Stevens-Johnson-Syndrom und toxisch epidermale Nekrolyse traditionell in das Spektrum des Erythema exsudativum multiforme (EEM) eingeordnet [2-4]. Dies mag darin begründet sein, dass kokardenförmige Hautveränderungen vorliegen und sich große Ähnlichkeiten in der Histologie der verschiedenen Reaktionsformen finden. Die Beschreibung des EEM - mit Schleimhautbeteiligung Erythema exsudativum multiforme majus (EEMM) - geht auf F. von Hebra (1866) zurück, auf den die späteren Autoren keinen Bezug nehmen. Basierend auf den o.g. Originalarbeiten wurde von einer internationalen Gruppe von Dermatologen eine Konsensusdefinition erarbeitet und publiziert.

\section{Konsensusdefinition}

Die Konsensusdefinition stützt sich auf die Beschreibung der Effloreszenzen in den Originalpublikationen über EEM, SJS und TEN sowie auf
VNR

2760512010047430472

Bibliografie

DOI http://dx.doi.org/

10.1055/s-0030-1255561

Akt Dermatol 2010; 36:

335-346 @ Georg Thieme

Verlag KG Stuttgart · New York

ISSN 0340-2541

Korrespondenzadresse

PD Dr. med.

\section{Maja Mockenhaupt}

Dokumentationszentrum schwerer Hautreaktionen (dZh)

Universitäts-Hautklinik

Hauptstr. 7

79104 Freiburg

dzh@uniklinik-freiburg.de 
Abb. 1 Erosive Lippenbeteiligung bei EEMM, SJS oder TEN.

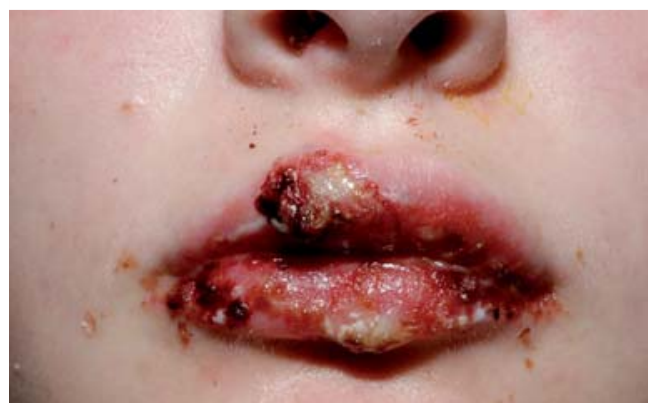

Abb. 2 Erosive Genitalschleimhaut bei EEMM, SJS oder TEN.
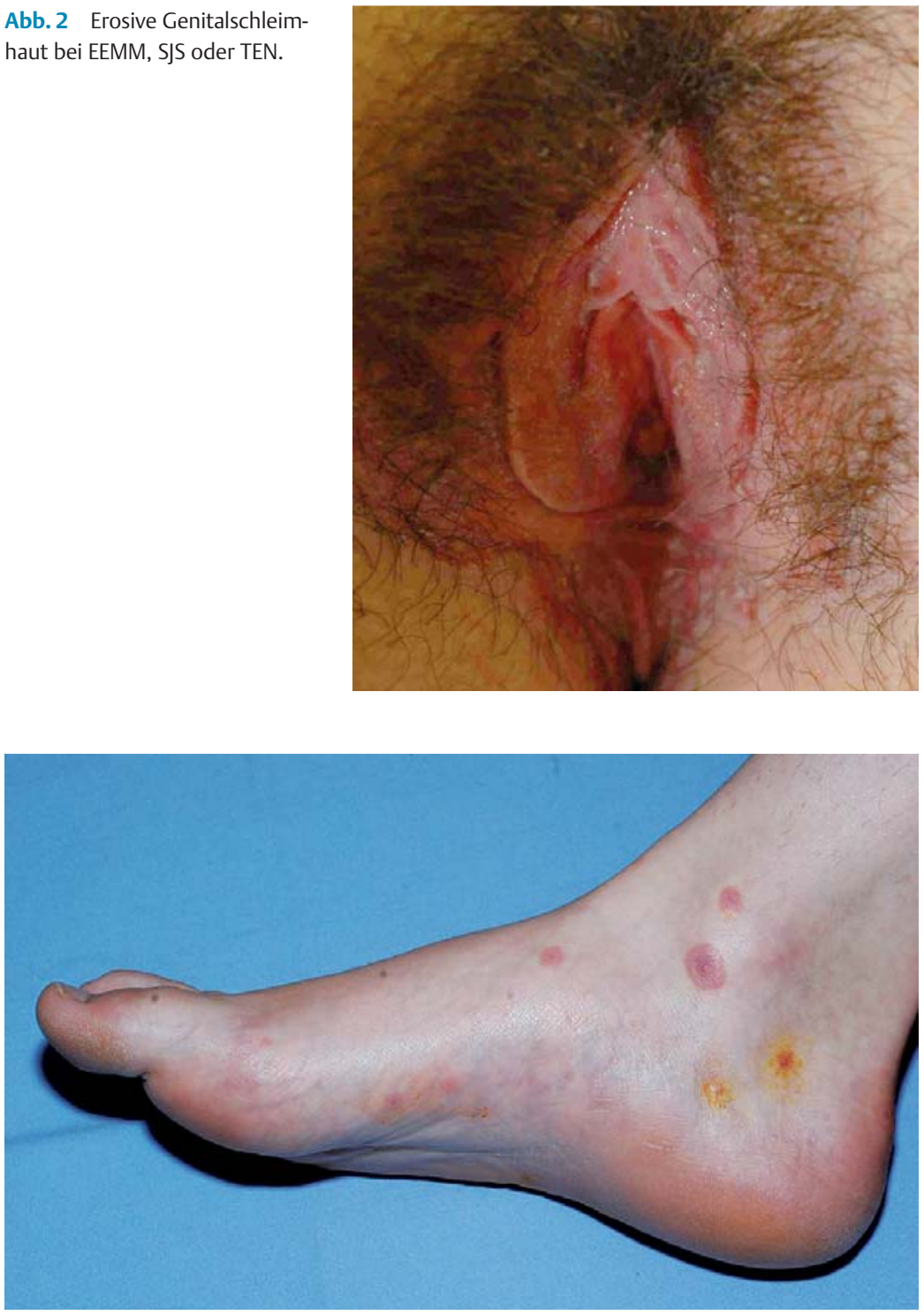

Abb. 3 Typische Kokarden am Fuß bei EEMM.

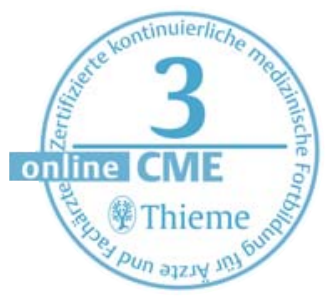

das Ausmaß an Blasenbildung bezogen auf die Körperoberfläche (KOF) [5].

Bei den Einzeleffloreszenzen werden sogenannte typische Kokarden („Schießscheiben-Läsionen“), welche aus 2 konzentrischen Ringen um ein erythematöses Zentrum bestehen und gegenüber der übrigen Haut klar abgegrenzt sind ( $\bullet$ Abb. 3), von atypischen Kokarden unterschieden.
Dabei sind atypische erhabene Kokarden eher unscharf begrenzt, zeigen einen weniger klaren zonalen Aufbau und imponieren infiltriert. Bei den atypischen flachen Kokarden fehlt das tastbare Ödem und auf kokardenähnlichen Erythemen findet sich häufig eine zentrale Blase. Bei den Maculae fehlt die Kokardenstruktur weitgehend, doch entwickeln sich auf ihnen ebenfalls Blasen.

Das SJS ist charakterisiert durch eine stammbetonte bzw. generalisierte Verteilung von eher atypischen Kokarden und Maculae, die konfluieren und auf denen Blasen entstehen, die dann quasi zusammenfließen ( $\bullet$ Abb. 4).

Die Hautablösung beschränkt sich dabei auf weniger als $10 \%$ der KOF. Der größte Prozentsatz an Hautablösung mit mehr als 30\% liegt bei der TEN mit Maculae vor ( $\bullet$ Abb. 5), wogegen der erosive Anteil bei der sehr seltenen Form der TEN auf großflächigen Erythemen (ohne Vorkommen kleiner konfluierender Flecke) wenig mehr als 10\% der KOF betrifft.

Da eine klare Abgrenzung zwischen SJS und TEN kaum möglich ist, wurde eine SJS/TEN-Übergangsform definiert mit Blasen und Erosionen zwischen $10 \%$ und 30\% der KOF. SJS, SJS/TENÜbergangsform und TEN werden als eine Krankheitsentität von verschieden schwerer Ausprägung angesehen, weshalb sie im Folgenden oft zusammenfassend als SJS/TEN bezeichnet werden. Im Gegensatz dazu imponiert das EEMM mit typischen Kokarden in extremitätenbetonter Verteilung ( Abb. 3; $\bullet$ Tab. 1) [5].

Erythema exsudativum multiforme majus (EEMM) und Stevens-Johnson-Syndrom (SJS) sind verschiedene Krankheitsbilder mit unterschiedlicher Ätiologie.

\section{Generalisiertes bullöses fixes Arzneiexanthem}

Das generalisierte bullöse fixe Arzneiexanthem (generalized bullous fixed drug eruption; GBFDE) kann klinisch, aber nicht histologisch von SJS und TEN unterschieden werden. Beim GBFDE imponieren bis zu hühnereigroße, bräunlich livide Flecke, auf denen schlaffe Blasen entstehen. Die Blasenbildung beträgt meist nur wenige Prozent der KOF und zwischen den oftmals großen Blasen bestehen große Areale intakter Haut ( $\bullet$ Abb. 6) [6]. Allerdings kann es bei wiederholtem Auftreten des GBFDE zu immer ausgedehnteren Hautablösungen und damit auch zu einem schweren Krankheitsbild kommen. Eine erosive Schleimhautbeteiligung besteht selten, und dann in eher blandem Ausmaß. Fieber und Krankheitsgefühl fehlen meist, und die Patienten befinden sich in einem deutlich besseren Allgemeinzustand als Patienten mit SJS oder TEN. In den meisten Fällen lässt sich anamnestisch eine frühere, meist lokalisierte Reaktion im Sinne eines fixen Arzneiexanthems eruieren $[1,7]$. 
Tab. 1 Konsensusdefinition der schweren blasenbildenden Hautreaktionen [5].

\begin{tabular}{|c|c|c|c|c|c|}
\hline Einteilung & EEMM & SJS & $\begin{array}{l}\text { SJS/TEN- } \\
\text { Übergangsform }\end{array}$ & $\begin{array}{l}\text { TEN mit Maculae } \\
\text { (Flecke) }\end{array}$ & $\begin{array}{l}\text { TEN auf großflächigen } \\
\text { Erythemen (ohne Flecke) }\end{array}$ \\
\hline Hautablösung & $<10 \%$ & $<10 \%$ & $10-30 \%$ & $>30 \%$ & $>10 \%$ \\
\hline typische Kokarden & + & - & - & - & - \\
\hline atypische Kokarden & erhaben & flach & flach & flach & - \\
\hline $\begin{array}{l}\text { Maculae } \\
\text { (Flecke) }\end{array}$ & - & + & + & + & - \\
\hline Verteilung & extremitätenbetont & $\begin{array}{l}\text { stammbetont/ } \\
\text { generalisiert }\end{array}$ & $\begin{array}{l}\text { stammbetont/ } \\
\text { generalisiert }\end{array}$ & $\begin{array}{l}\text { stammbetont/ } \\
\text { generalisiert }\end{array}$ & stammbetont/generalisiert \\
\hline
\end{tabular}

Das generalisierte bullöse fixe Arzneimittelexanthem lässt sich klinisch von SJS und TEN unterscheiden und die Patienten haben eine bessere Prognose.

\section{Diagnostische Maßnahmen}

$\nabla$

\section{Klinische Zeichen}

Die Verdachtsdiagnose einer schweren bullösen Hautreaktion wird primär klinisch gestellt. Zu Beginn der Erkrankung ist die Einordnung des Exanthems bei SJS und TEN schwierig, vor allem wenn noch keine Schleimhautbeteiligung vorliegt. Auch wenn bereits Blasen und Erosionen der Haut vorhanden sind, lässt sich kaum abschätzen, ob das Maximum der Reaktion bereits erreicht ist oder ob die Hautablösung weiter voranschreitet. Ein wichtiges klinisches Zeichen bei SJS und TEN sowie deren Übergangsform besteht darin, dass sich die frischen Blasen quasi verschieben lassen bzw. sich die Epidermis auf noch nicht bullös veränderter Haut wegschieben lässt, d. h. das Nikolski-Phänomen ist positiv. Bei GBFDE hingegen ist das Nikolski-Phänomen in den nicht blasigen gesunden Hautarealen negativ [8]. Spezifische Laborparameter zur Diagnosesicherung von SJS, SJS/TEN-Übergangsform, TEN oder GBFDE stehen nicht zur Verfügung.

Spezifische Laborparameter zur Diagnosesicherung von SIS/TEN gibt es nicht.

\section{Probebiopsie und Histologie}

Die Schnellschnittdiagnostik eines Kryostatpräparates kann eine rasche Differenzierung zwischen subepidermaler und intraepidermaler Spaltbildung ermöglichen. Letztere kommt beim Staphylococcal scalded Skin Syndrome (SSSS) vor, welches durch ein Staphylokokken-Exotoxin ausgelöst wird und früher auch „staphylogenes Lyell-Syndrom“ genannt wurde $[9,10]$. Um möglichst rasch die Höhe der epidermalen Spaltbildung zu bestimmen und damit zwischen SJS/TEN und SSSS zu unterscheiden, kann mit dem sog. Tzanck-Test eine exfoliative Zytologie durchgeführt werden. Beim SSSS finden sich breite epitheliale Zellen mit einer kleinen Kern-Zytoplasma-Relation, wohingegen sich bei der TEN kuboidale Zellen mit großer Kern-Zytoplasma-Relation

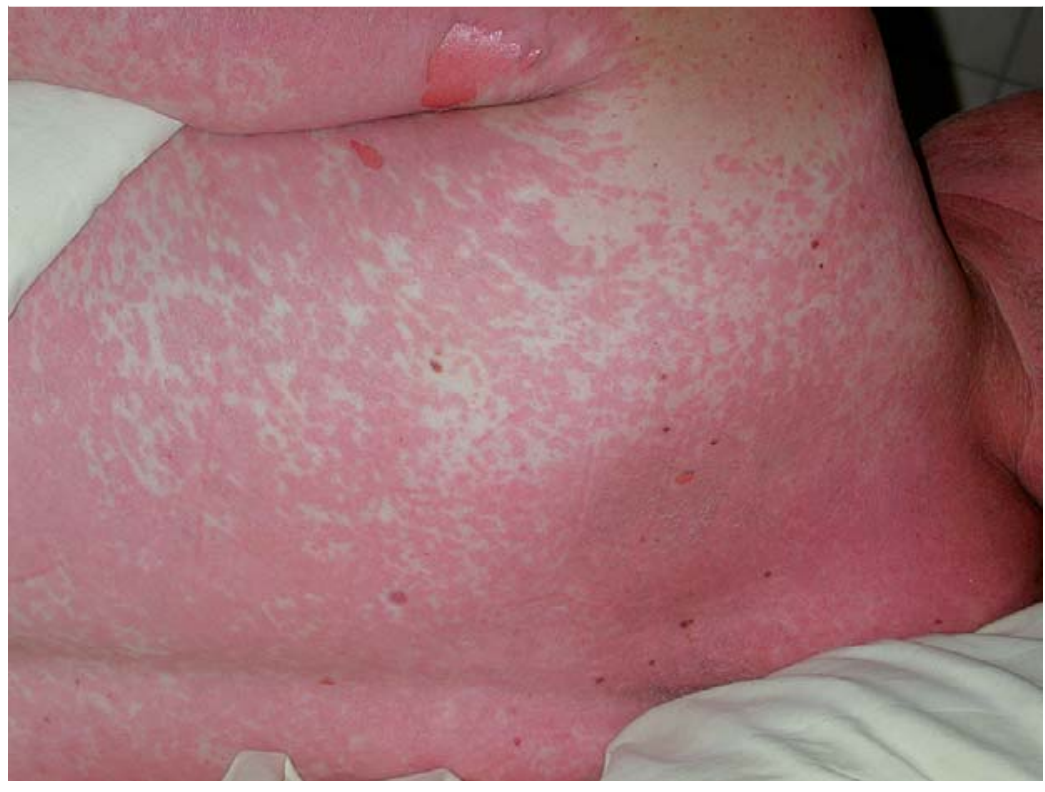

Abb. 4 Atypische Kokarden und Maculae bei SJS.

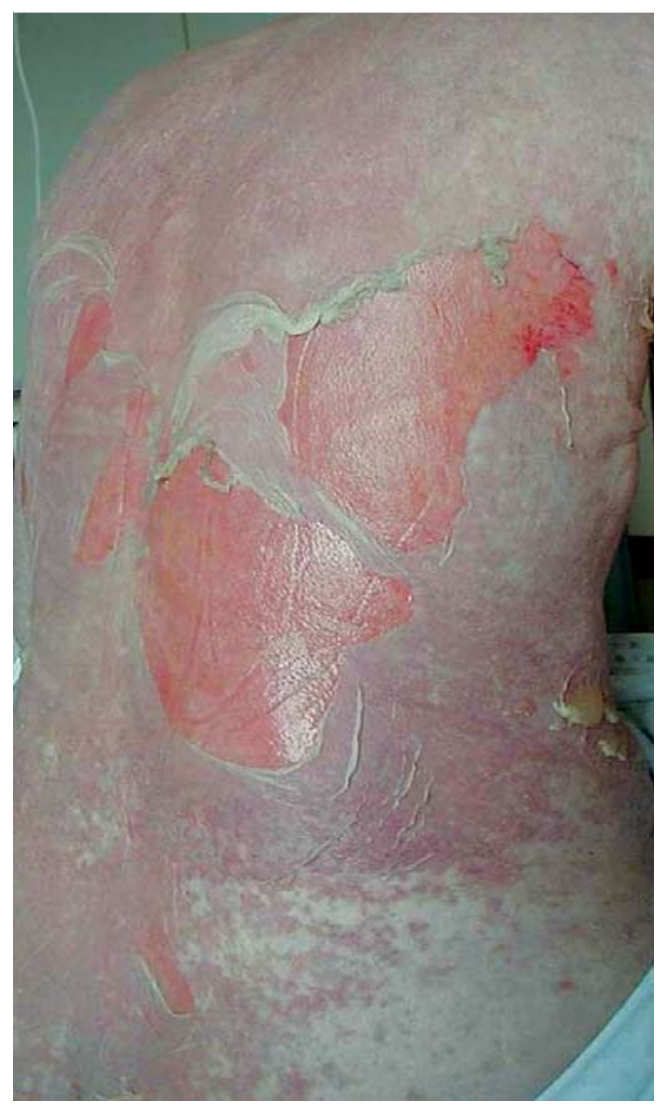

Abb. 5 Großflächige Hautablösung bei TEN.

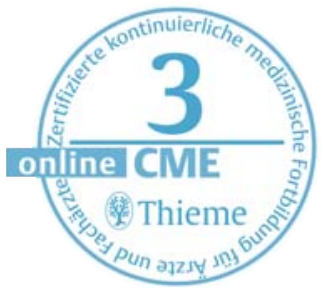




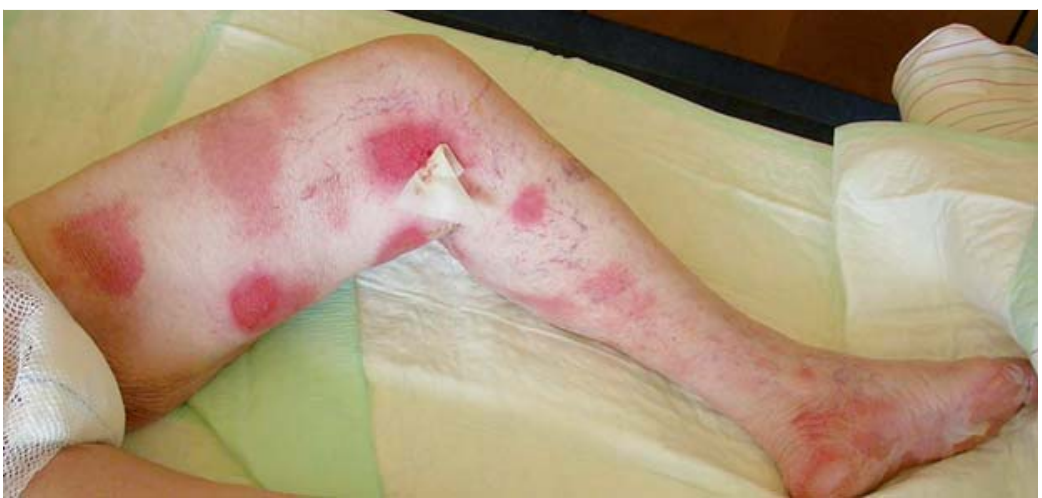

Abb. 6 Einzelstehende große Blasen bzw. Erosionen bei GBFDE.

nachweisen lassen. Dennoch sollte möglichst bei allen Fällen von schweren Hautreaktionen eine Probebiopsie zur konventionellen histologischen Aufarbeitung entnommen werden. Am besten entnimmt man die Probe aus dem erythematösen Randbereich der blasigen Läsionen. Hierdurch gelingt es in der Regel, sowohl Epidermis- als auch Dermisanteile im Biopsat zu gewinnen. Bei SJS und TEN, wie auch bei GBFDE, zeigt die Histologie nekrotische Keratinozyten, die in disseminierter Verteilung vorliegen oder aber zu einer kompletten Nekrose der Epidermis führen. Daneben findet sich eine Vakuolisierung der Basalmembranzone bis hin zur subepidermalen Spalte. In der oberen Dermis imponiert ein perivaskuläres, lymphohistiozytäres Infiltrat, in dem auch eosinophile Granulozyten vorkommen [11]. Die beim fixen Arzneiexanthem beschriebene Pigmentablagerung in tieferen Hautschichten fehlt beim akuten GBFDE häufig, weshalb die histologische Abgrenzung zu SJS/TEN kaum möglich ist. Falls differenzialdiagnostisch autoimmunologische blasenbildende Dermatosen, wie z.B. Pemphigus vulgaris, bullöses Pemphigoid oder IgA-lineare Dermatose in Betracht gezogen werden, so sollte eine Immunfluoreszenz-Untersuchung des Hautbiopsats erfolgen.

Bei Verdacht auf eine schwere Hautreaktion sollte immer eine Probebiopsie entnommen und ggf. eine Immunfluoreszenz-Untersuchung durchgeführt werden.

\section{Differenzialdiagnosen \\ $\nabla$}

Erythema exsudativum multiforme majus Die wichtigste Differenzialdiagnose des StevensJohnson-Syndroms ist das Erythema exsudativum multiforme majus (EEMM), bei welchem sich typische oder atypische, erhabene Kokarden vorwiegend im Bereich der Extremitäten bei gleichzeitigem Vorliegen einer erosiven Schleimhautbeteiligung finden ( $\bullet$ Abb. 3). Häufig werden die Minor- wie auch die Majorform des EEM durch akute oder rezidivierende Eruptionen von Herpes simplex induziert. Auch Mykoplasmenpneumonien, Infekte der oberen Luftwege, Influenza und grippale Infekte kommen als Auslöser des EEMM infrage. Somit gelten EEM und EEMM nicht als kutane Arzneimittelreaktionen, weshalb sie vom SJS unterschieden werden sollten [12]. Dies gilt auch für untypische Formen des EEMM bei Kindern und Jugendlichen, die typische Kokarden und/ oder atypische „Riesenkokarden“ in stammbetonter oder generalisierter Verteilung aufweisen. Bei einem EEMM mit extremitätenbetonter Verteilung und gut abgegrenzten Kokardenläsionen ist nicht von einem möglichen Übergang in ein konfluierendes Exanthem mit großflächiger Blasenbildung im Sinne einer TEN auszugehen, was nicht nur therapeutisch, sondern auch hinsichtlich der Prognose des Patienten von Bedeutung ist. Die erosiven Schleimhautveränderungen kommen bei allen Formen der schweren Hautreaktionen in gleicher Weise vor, was bedeutet, dass die verschiedenen Reaktionsformen nicht aufgrund ihrer Schleimhautläsionen unterschieden werden können.

EEMM wird in erster Linie infektiös getriggert, bei Erwachsenen v. a. durch Herpes simplex-Eruptionen, bei Kindern und Jugendlichen häufiger durch Mykoplasmeninfektionen und Infekte der oberen Luftwege. SJS hingegen wird überwiegend durch Arzneimittel ausgelöst, ebenso wie TEN.

\section{Weitere Differenzialdiagnosen}

Zu den weiteren Differenzialdiagnosen des SJS gehören makulöse und vesikulöse Exantheme verschiedener Genese, wobei gerade bei Kindern Virusexantheme oft mit einer Schleimhautbeteiligung einhergehen. Ausgeprägte Arzneimittelexantheme, die manchmal einen multiformen, kokardenförmigen Aspekt haben, keine Schleimhauterosionen aufweisen und histologisch abgrenzbar sind, sollten ebenfalls in die differenzialdiagnostischen Überlegungen einbezogen werden [13].

Die wichtigsten Differenzialdiagnosen der TEN sind das generalisierte bullöse fixe Arzneiexanthem (generalized bullous fixed drug eruption; GBFDE) und das Staphylococcal scalded Skin Syndrome (SSSS). Das klinische Bild von GBFDE unterscheidet sich von SJS und TEN, wie bereits oben beschrieben, während der histologische Befund einer aus einem blasigen Areal entnommenen Probebiopsie keine Unterscheidung erlaubt. Im Gegensatz zum fleckigen, konfluierenden Exanthem bei SJS und TEN findet sich beim SSSS ein großflächiges, zum Teil in eine Erythrodermie übergehendes Erythem. Das Nikolski-Phänomen ist häufig positiv, die Hautablösung aber sehr oberflächlich [8]. Die Histologie beim SSSS weist eine intraepidermale, subkorneale Blasenbildung auf. Eine Schleimhautbeteiligung ist beim SSSS nur diskret vorhanden. Das SSSS ist insgesamt deutlich seltener als SJS und TEN und zeigt eine unterschiedliche Altersverteilung mit einem Altersgipfel im Kleinkindes- und einem im Erwachsenenalter [10]. 
Daneben müssen autoimmunologisch bedingte blasenbildende Erkrankungen wie der Pemphigus vulgaris und das bullöse Pemphigoid, aber auch bullöse phototoxische Reaktionen als mögliche Differenzialdiagnosen von SJS und TEN in Betracht gezogen werden. Ebenso sind ausgeprägte Arzneimittelexantheme sowie Erythrodermien differenzialdiagnostisch zu erwägen. Vor allem die großflächige Abschuppung bei der exfoliativen Dermatitis kann manchmal mit der epidermalen Hautablösung bei TEN verwechselt werden. Bei der akuten generalisierten exanthematischen Pustulose (AGEP) kann es durch Konfluieren der Pusteln zur intraepidermalen Hautablösung kommen, die ein positives Nikolski-Phänomen vortäuscht. Sowohl bei AGEP als auch beim Hypersensitivitätssyndrom (HSS), heute meist als „Drug reaction with Eosinophilia and systemic Symptoms“ (DRESS) bezeichnet, können vereinzelt kokardenartige Hautveränderungen und leichte Schleimhautläsionen auftreten, die v.a. zu Beginn der Reaktion den Verdacht auf SJS oder TEN nahe legen. Bei HSS/DRESS kommt es nicht selten zum Auftreten von Spannungsblasen aufgrund des ausgeprägten Ödems der Haut, die an SJS und TEN denken lassen $[14,15]$.

\section{Ätiologie}

\section{Pharmakoepidemiologische Daten}

Die Risikobewertung von Arzneimitteln zur Auslösung von schweren blasenbildenden Hautreaktionen stützt sich in erster Linie auf klinisch-epidemiologische Daten, denn es gibt kein In-vivooder In-vitro-Testverfahren, welches das auslösende Agens von SJS und TEN sicher identifizieren könnte. Hauttestungen wie z.B. Epikutantestungen und Reexpositionstestungen mit dem vermuteten Auslöser sind häufig negativ verlaufen. Eine zwischen 1997 und 2001 durchgeführte europäische Fall-Kontrollstudie konnte die bereits früher beschriebenen hohen Risiken zur Auslösung von SJS und TEN für folgende Substanzen bestätigen: Allopurinol, antibakterielle Sulfonamide, nichtsteroidale Antirheumatika vom Oxicamtyp und verschiedene Antiepileptika (Carbamazepin, Phenobarbital, Phenytoin) [16]. Das hohe Risiko von Allopurinol wurde in erster Linie in Deutschland beobachtet, wobei in den meisten Fällen keine manifeste Gicht, sondern lediglich eine diskrete, asymptomatische Hyperurikämie zur Verordnung der Substanz führte. Dies sollte zum umsichtigeren Umgang und zur engeren Indikationsstellung für die Therapie mit Allopurinol führen $[17,18]$. Folgende neuere Arzneimittel konnten als Risikofaktoren für SJS und TEN identifiziert werden: das Antiepileptikum Lamotrigin und der non-nucleoisidische Reverse-Transkriptaseinhibitor Nevirapin.

\section{Potenziell SJS|TEN-auslösende Medikamente}

Praktische Empfehlungen zur Beurteilung [16]

- Einige Arzneimittel besitzen ein hohes Risiko zur Auslösung von SJS oder TEN. Vor deren Verordnung sollte der erwartete Nutzen sorgfältig evaluiert werden. Dies gilt für:

- Nevirapin

- Lamotrigin

- Carbamazepin

- Phenytoin

- Phenobarbital

- Cotrimoxazol und andere antibakterielle Sulfonamide

- Sulfasalazin

- Allopurinol

- nicht-steroidale Antirheumatika vom Oxicamtyp

Eine Latenz von 4 Tagen bis 4 Wochen zwischen Beginn der Medikamenteneinnahme und Auftreten der schweren Hautreaktion entspricht dem zeitlichen Muster für „hochverdächtige“ Auslöser und spricht für einen kausalen Zusammenhang.

Wenn gleichzeitig mehrere Arzneimittel eingenommen werden, spielt der Zeitraum der Einnahme eine große Rolle für die Entscheidung, welche Substanz(en) abgesetzt werden muss (müssen) und ob einige weiterhin eingenommen oder erneut angesetzt werden können.

Das Risiko von verschiedenen Antibiotika zur Auslösung von SJS und TEN liegt etwa in derselben Größenordnung, aber deutlich unter dem Risiko der antibakteriellen Sulfonamide.

- Valproinsäure scheint im Gegensatz zu einigen anderen Antiepileptika kein erhöhtes Risiko für schwere Hautreaktionen zu haben.

Diuretika und orale Antidiabetika mit Sulfonamid-ähnlicher Struktur stellen keine Risikofaktoren für SJS und TEN dar.

Die durchschnittliche Zeitspanne zwischen Einnahmebeginn des Medikaments und Auftreten von SJS oder TEN beträgt für die meisten Substanzen mehrere Tage bis Wochen [16]. Während das Risiko für Lamotrigin etwa dem der o.g. anderen Antiepileptika vergleichbar ist, liegt das Risiko zur Auslösung von SJS und TEN bei Nevirapin deutlich höher als bei anderen zur HIV-Therapie eingesetzten Substanzen. In Mitteleuropa sind knapp 5\% der SJS/TEN-Patienten mit HIV infiziert, doch die HIV-Infektion scheint kein unabhängiger Risikofaktor für SJS und TEN zu sein [19]. In einer epidemiologischen Studie konnte festgestellt werden, dass jeweils etwa 65\% der Patienten mit SJS, TEN und deren Übergangsformen im relevanten Zeitraum vor der Reaktion ein Arzneimittel eingenommen hatten, das als hochverdächtiger Auslöser von schweren Hautreaktionen bekannt war [16]. In etwa einem Viertel der Fälle von SJS/ TEN lässt sich kein medikamentöser Auslöser festmachen. Teilweise findet sich ein Infektgeschehen im relevanten Zeitraum vor Beginn der Hautreaktion, welches als Auslöser in Frage kommt, andere Fälle müssen als idiopathisch betrachtet werden.

Für GBFDE liegen keine Daten aus epidemiologischen Untersuchungen vor, sondern lediglich gröBere Fallserien [6,7]

Während es sich bei SJS und TEN in der Regel um den ersten Einnahmezyklus eines Arzneimittels handelt, also keine vorherige Sensibilisierung vorliegt, lässt sich beim GBFDE fast immer ein entsprechendes, wenn auch weniger ausgeprägtes Vorereignis, meist auf dasselbe Arzneimittel eruieren. Bei genauer Anamnese erfährt man nicht selten, dass die erste Einnahme des Arzneimittels gut vertragen wurde und erst die erneute Einnahme zur Unverträglichkeitsreaktion führte, die bei

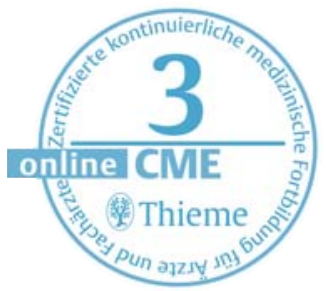




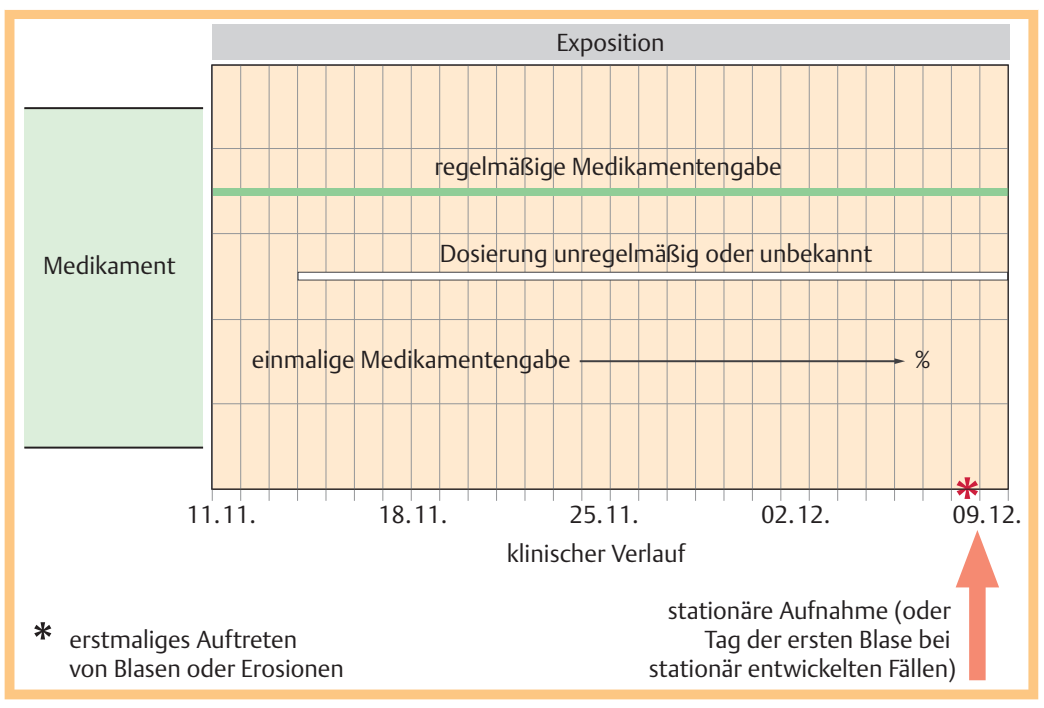

Abb. 7 Zeitstrahldiagramm zur Darstellung der Medikamentenanamnese.

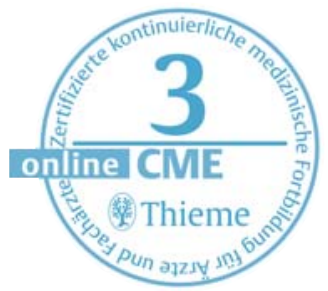

wiederholter Einnahme ausgeprägter verlaufen kann. Als häufigster Auslöser des GBFDE konnte in Deutschland Cotrimoxazol identifiziert werden, wobei die zeitliche Latenz zwischen Einnahmebeginn und Beginn der Hautreaktion oft nur mehrere Stunden bis wenige Tage beträgt [7].

\section{Kausalitätsbewertung im Einzelfall}

Im Einzelfall basiert die Identifizierung des auslösenden Arzneimittels vor allem auf dem Zeitintervall zwischen Beginn der Einnahme des Arzneimittels und Beginn der schweren Hautreaktion, welches bei SJS und TEN meist bei etwa 1-4 Wochen liegt. Zudem spielt eine Rolle, ob das verdächtige Arzneimittel als Auslöser schwerer Hautreaktionen bekannt ist und ein erhöhtes Risiko in pharmakoepidemiologischen Studien festgestellt wurde.

Um das auslösende Arzneimittel identifizieren und absetzen zu können, muss eine sehr detaillierte und gründliche Arzneimittelanamnese erhoben werden. Hierzu eignet sich die Erstellung eines Zeitstrahldiagramms, in das einerseits die klinischen Symptome (x-Achse) und andererseits die eingenommenen oder applizierten Arzneimittel (y-Achse) eingetragen werden können ( $\bullet$ Abb. 7). Oftmals sind die Angaben der in ihrem Allgemeinbefinden stark beeinträchtigten Patienten nicht ausreichend, sodass weitere Informationsquellen über mögliche verordnete und/oder eingenommene Arzneimittel herangezogen werden müssen. Es ist für die betroffenen Patienten sehr wichtig, das/die auslösende/n Arzneimittel zu identifizieren oder zumindest möglichst einzugrenzen. Ein entsprechender Allergiepass sollte ausgestellt werden. Geschieht das nicht, haben viele Patienten Angst, überhaupt jemals wieder ein Arzneimittel einzunehmen, was sich negativ auf ihre Gesundheit auswirken kann.

\section{Pathogenese}

Pathogenetische Überlegungen gingen schon früh von einem veränderten Arzneimittelmetabolismus bei Patienten mit Stevens-Johnson-Syndrom und toxisch epidermaler Nekrolyse aus. Der Vergleich der Acetylierungskapazität in einer FallKontrollstudie zeigte, dass die durchschnittliche Aktivität der N-Acetyltransferase bei Patienten mit SJS und TEN signifikant niedriger war als bei gesunden Kontrollpersonen. Obwohl der Genotyp der Langsam-Acetylierer vor allem bei Patienten mit Sulfonamid-induzierten schweren Hautreaktionen beschrieben wurde, hatten die hier untersuchten Patienten eine Vielzahl verschiedener Arzneimittel eingenommen, von denen $26 \%$ via Acetylierung metabolisiert werden [20].

SJS und TEN werden als immunologisch mediierte Reaktionsformen angesehen, wobei immunhistochemisch in der Dermis CD4+-Zellen, in der Epidermis CD8+-Zellen überwiegen. Daher fasst man diese schweren Hautreaktionen als T-Zellvermittelte Reaktion auf, vergleichbar einer Graft-versus-Host-Erkrankung (GvH). Die akute Nekrose der Keratinozyten bei SJS und TEN wird dabei auf einen ausgedehnten apoptotischen Prozess zurückgeführt. Zytotoxische T-Zellen können Apoptose initiieren, verstärkt durch das Freisetzen von Perforinen und Zytokinen, wie z.B. Tumornekrosefaktor- $\alpha$ (TNF- $\alpha$ ) oder Granzym B. Weiterhin wird angenommen, dass Proteine wie FAS-Antigen (CD 95) und P55-TNF- $\alpha$-Rezeptor die Apoptose in Keratinozyten fördern [21]. Allerdings konnte mittlerweile gezeigt werden, dass nicht FAS und FAS-Ligand die wichtigsten Zytokine in der Akutphase von SJS und TEN sind, sondern das kationische Protein Granulysin. Es wies in der Blasenflüssigkeit von Patienten mit SJS/ TEN die stärkste Zytotoxizität auf, wobei seine Konzentration mit der Schwere des Krankheitsbildes zunahm. Bei anderen blasenbildenden Hauterkrankungen hingegen konnte Granulysin nicht in der Blasenflüssigkeit nachgewiesen werden [22]. Daraus ließe sich der Schluss ziehen, dass Granulysin ein Marker für den Schweregrad von SJS/TEN ist und eine Angriffsfläche für potenzielle Therapien bietet.

Die Keratinozytennekrose mit nachfolgender Epidermisablösung bei SJS/TEN wird auf einen apoptotischen Prozess zurückgeführt.

Obwohl schon früher eine genetische Prädisposition bei Patienten mit schweren Hautreaktionen vermutet wurde [23], ließ sich erst in neueren $\mathrm{Ar}$ beiten zeigen, dass diese Prädisposition spezifisch für bestimmte Arzneimittel ist und starke ethnische Unterschiede aufweist. So konnte z.B. der hochsignifikante Zusammenhang zwischen Carbamazepin-induziertem/r SJS bzw. TEN und HLAB*1502 bei Han-Chinesen bei europäischen Patienten nicht bestätigt werden [24,25]. Bei Allo- 
purinol-induziertem/r SJS bzw. TEN hingegen konnte HLA-B*5801 sowohl bei Han-Chinesen (100\%) als auch bei Europäern (55\%) nachgewiesen werden $[26,27]$. SJS und TEN sind offenbar sehr arnzeimittelspezifische Reaktionsformen, d.h. ein bestimmter genetisch prädisponierter Patient reagiert nur auf ein spezifisches Arzneimittel mit SJS/TEN. Dieses sollte er zur Sicherheit meiden, auch wenn frühere Testreihen bei Reexposition nur in etwa 10\% (im Gegensatz zu GBFDE) zu einer erneuten Reaktion führten [6].

Eine arzneimittelspezifische genetische Disposition legt nahe, dass ein Patient nur auf ein Arzneimittel reagiert.

Beim GBFDE ist aber nicht nur mit einer positiven Reaktion bei Reexpositionstestung zu rechnen, sondern auch Epikutantestungen mit dem verdächtigen Agens sind häufig positiv, v.a. wenn in loco getestet wird, d.h. im Bereich der fixen Arzneimittelexantheme. Dies scheint auf dem Vorliegen residualer T-Zellen (effector memory T-cells) zu basieren [28].

\section{Therapie}

\section{Allgemeine Maßnahmen}

Da die Pathogenese von SJS und TEN nicht abschließend aufgeklärt ist, beschränkt sich ihre Therapie auf symptomatische und unspezifische Maßnahmen. Arzneimittel, die im Verdacht stehen, die schwere Hautreaktion ausgelöst zu haben, sollten nach Erheben einer detaillierten Medikamentenanamnese abgesetzt werden. Dies sind vor allem Substanzen, die innerhalb von 3-4 Wochen vor Beginn der Hautreaktion neu angesetzt wurden. Wenn die Hautablösung mehr als $30 \%$ der Körperoberfläche beträgt (entsprechend etwa einer Epidermisablösung des gesamten Stammes ventral und dorsal), ist es ratsam, die betroffenen Patienten auf eine Intensivstation oder in eine Verbrennungseinheit zu verlegen, zumindest wenn internistische Grunderkrankungen vorliegen und entsprechende Überwachung geboten scheint. Zu den symptomatischen Maßnahmen gehören die Erhöhung der Raumtemperatur auf $30-32^{\circ} \mathrm{C}$, die Lagerung auf Metalline-Folie bzw. in einem Luftkissenbett (z. B. Clinitron ${ }^{\circledR}$ ) und die intravenöse Flüssigkeitszufuhr mit z.B. Albuminlösung oder isotonischer Elektrolytlösung entsprechend intensivmedizinischer Behandlungsprotokolle. Dabei sollte bedacht werden, dass Patienten mit TEN einen gegenüber Verbrennungspatienten verminderten Flüssigkeitsbedarf haben, der bei etwa 2 Dritteln bis 3 Vierteln des Bedarfs von Verbrennungsopfern liegt. Einige Spezialisten schlagen vor, kolloidale Infusionslösungen zu vermeiden, und empfehlen stattdessen die Infusion von Ringerlösung bis zu einer renalen Ausscheidung von $2 \mathrm{ml}$ pro kg Körpergewicht pro
Stunde. Um den Proteinverlust zu minimieren, aber auch um eine intestinale Atonie zu vermeiden, ist es ratsam, eine frühzeitige Ernährung über eine Nasensonde zu beginnen. Dabei wird vorgeschlagen, 1500 Kalorien in 1500 ml Flüssigkeit in den ersten 24 Stunden zu applizieren; danach sollte sich die Energiezufuhr um etwa 500 Kalorien pro Tag bis zu 4000 Kalorien pro Tag steigern [29].

Von einer breiten antibiotischen Abdeckung ohne Verdacht auf eine Infektion, d.h. von einer rein prophylaktischen Gabe, sollte man absehen, v.a. weil so Infektzeichen verschleiert werden können. Dagegen sollten Antibiotika gezielt dann eingesetzt werden, wenn Zeichen einer Infektion oder Septikämie vorliegen, wobei die jeweilige Substanz entsprechend der Sensitivität der vorhandenen Erreger auszuwählen ist.

Supportive Maßnahmen sind bei Patienten mit SJS/TEN vorrangig und richten sich im Wesentlichen nach dem Ausmaß der Hautablösung. Dieses sollte korrekt bestimmt werden, was v. a. bei Vorliegen vieler Einzelläsionen schwierig sein kann und häufig zur Überschätzung führt.

\section{Lokaltherapie}

Hinsichtlich der Lokalbehandlung werden antiseptische Lösungen oder Gele empfohlen (z.B. Octenidin, Chlorhexidin, Silbernitrat); auf belastete Körperareale kann wirkstofffreie, nicht klebende Netzgaze aufgebracht werden. Aus dermatologischer Sicht wird von der Anwendung sulfonamidhaltiger Externa abgeraten, da Sulfonamide einen bekannten Risikofaktor für die Entstehung von schweren Hautreaktionen darstellen. Zum Teil noch pralle Blasen können aseptisch geöffnet werden, wobei die nekrotische Epidermis belassen wird, um ein zu starkes Austrocknen der Haut zu verhindern und so die Reepithelisierung zu fördern [29].

Von Verbrennungsmedizinern hingegen wird das Entfernen der nekrotischen Epidermis bevorzugt, welches entweder durch ein silbernitrathaltiges sog. Verbrennungsbad oder durch agressives Bürstendébridement erfolgt. Nach Ablösen der Epidermis wird die sofortige Wundabdeckung mit biologischen Dressings (z.B. Biobrane ${ }^{\circledR}$ ) aber auch kutanen Allo- oder Xenotransplantaten vorgenommen. Das mag die Lagerung und Bewegung des Patienten erleichtern, doch birgt das Débridement das Risiko der Narbenbildung bei einer ansonsten nicht zur Vernarbung führenden subepidermalen Blasenbildung. Einige Verbrennungsmediziner gehen daher bereits restriktiver damit um [30].

\section{Aggressives Bürstendébridement und Festtackern von Wundauflagen in der Haut sollten vermieden werden, da dies zur Narbenbildung führen kann.}

Für die Behandlung der erosiven Mundschleimhautbeteiligung werden antiseptische Mundspü- 
Tab. 2 Unabhängige Prognosefaktoren für TEN-SCORTEN (Möglicher Score 0 - 7) [36].

\begin{tabular}{|c|c|c|}
\hline \multicolumn{3}{|l|}{ Variable } \\
\hline Alter ( $\geq 40$ Jahre) & ja & 1 \\
\hline Herzfrequenz ( $\geq 120 / \mathrm{min}$ ) & ja & 1 \\
\hline maligne Grunderkrankung & ja & 1 \\
\hline $\begin{array}{l}\text { Ablösung der Körperoberfläche am 1. Tag } \\
<10 \% \\
10-30 \% \\
>30 \%\end{array}$ & $\geq 10 \%$ & 1 \\
\hline Harnstoff im Serum ( $\geq 10 \mathrm{mmol} / \mathrm{l}$ ) & ja & 1 \\
\hline Bicarbonat im Serum (<20 mmol/l) & ja & 1 \\
\hline Glukose im Serum ( $\geq 14 \mathrm{mmol} / \mathrm{l})$ & ja & 1 \\
\hline
\end{tabular}

lungen empfohlen, bei betroffenen Lippen verschaffen dexpanthenolhaltige Salben Linderung. Auch für die Behandlung von genitalen oder analen Erosionen kommen antiseptische Lösungen und Cremes zur Anwendung. Äußerst wichtig ist eine ophthalmologische Betreuung bei Patienten mit Augenbeteiligung in Form einer Konjunktivitis oder Blepharitis. Okuläre Schleimhautläsionen erfordern eine tägliche Untersuchung durch einen erfahrenen Augenarzt. Neben antiseptischen oder antibiotischen Augentropfen muss bei vielen Patienten eine Symblepharon-Prophylaxe durch konsequente Lidrandpflege oder das Einlegen von Illigschalen in Betracht gezogen werden, um mögliche schwere Folgeschäden zu vermeiden. Spätere chirurgische Maßnahmen, wie das Lösen von Synechien, sind äußerst problematisch. Auch Schleimhauttransplantate, wie z.B. Konjunktivenersatz durch Mundschleimhautanteile oder Transplantation von Limbuszellen, können das Problem nicht endgültig lösen. Bei Patienten, die ein Sicca-ähnliches Syndrom entwickeln, können künstliche Tränenflüssigkeiten eine Besserung erzielen [31].

Die augenärztliche Mitbetreuung von Patienten mit SJS/ TEN ist extrem wichtig, da Folgeschäden im Bereich der Augen langfristige und schwierige Therapien nach sich ziehen können.

\section{Systemische immunmodulierende \\ Therapie}

Da SJS und TEN als immunologisch vermittelte Unverträglichkeitsreaktionen anzusehen sind, werden häufig Glukokortikosteroide zur Therapie von SJS und TEN eingesetzt. Der Nutzen und das Risiko dieser Behandlung werden weltweit kontrovers diskutiert, ähnlich wie bei anderen immunmodulierenden Therapien einschließlich Cyclophosphamid, Cyclosporin oder intravenösen Immunglobulinen (IVIG). Thalidomid, welches als effektiver TNF- $\alpha$-Blocker erfolgreich zur Behandlung der GvHD eingesetzt wird, erwies sich in der Therapie von TEN als schädlich und darf für diese Indikation nicht verwendet werden [32]. Über den Einsatz neuerer TNF- $\alpha$-Antagonisten zur Behandlung von SJS und TEN liegen bislang nur Einzelfallberichte vor, die keine zuverlässige Bewertung erlauben. IVIG werden zur Therapie von SJS und TEN eingesetzt, da Antikörper in gepoolten humanen Immunglobulinen in vitro die FAS-mediierte Keratinozytennekrosen blockieren [33]. Auch hier gibt es widersprüchliche Studienergebnisse, wobei eine Reihe von Patienten mit positivem Krankheitsausgang nach IVIG-Therapie wiederholt in verschiedenen, meist retrospektiven Studien und Fallsammlungen auftauchen [34]. In einer prospektiven Untersuchung hingegen wurde gezeigt, dass weder die Progression der Erkrankung gestoppt noch die Reepithelisierung befördert werden konnte. Zudem war die Letalität höher als erwartet, verglichen mit dem zur Evaluierung der Prognose entwickelten SCORTEN [35]. Dabei handelt es sich um ein Instrument zur Bewertung des Schweregrades von SJS und TEN, welches innerhalb der ersten Tage im Verlauf der Reaktion angewendet werden sollte. Jeder der 7 unabhängigen Faktoren erhält das gleiche Gewicht, wobei die Überlebenschance für den betroffenen Patienten mit Zunahme der Scorewerte fällt ( Tab. 2) [36].

Eine große retrospektive Untersuchung zur Therapie von SJS und TEN, welche in Frankreich und Deutschland durchgeführt wurde, konnte zeigen, dass Glukokortikosteroide in mittlerer bis hoher Dosierung (100-500 mg), initial und kurzzeitig gegeben, den Ausgang der schweren Hautreaktion positiv beeinflussen. Dies konnte für IVIG nicht nachgewiesen werden [37].

Derzeit gibt es keine immunmodulierende Standardtherapie von SIS/TEN. Im Einzelfall muss entschieden werden, ob der Patient über die supportive Behandlung hinaus von einer immunmodulierenden Therapie profitieren kann.

Zur Behandlung von ausgedehnten Formen des generalisierten bullösen fixen Arzneiexanthems können systemisch Steroide eingesetzt werden, meist ist dies aber nicht notwendig. Die topische Behandlung der lokalisierten Blasen und Erosionen sollte ähnlich wie bei SJS und TEN durchgeführt werden. Gleiches gilt für die manchmal vorliegende, aber vergleichsweise milde Schleimhautbeteiligung. Die wichtigste Maßnahme ist auch hier das Erkennen und Absetzen des auslösenden Arzneimittels.

Da sich die Hautveränderungen im Verlauf der Erkrankung wandeln und sehr unterschiedlich präsentieren können, empfiehlt sich bei allen Arzneimittelreaktionen der Haut eine gute Fotodokumentation zu Beginn und im Verlauf der Erkrankung.

\section{Prognose und Folgeschäden}

\section{$\nabla$}

Die Prognose von Patienten mit ausgedehnten bullösen Arzneimittelreaktionen ist insgesamt ungünstig, hängt aber neben dem Ausmaß der Hautablösung wesentlich vom Alter und den bereits bestehenden Krankheiten ab. Zwar können schwere Hautreaktionen in jedem Lebensalter 
auftreten, doch zeigt sich anhand der im Dokumentationszentrum schwerer Hautreaktionen (dZh) populationsbezogen in Deutschland erhobenen Daten, dass das Lebensalter der betroffenen Patienten ansteigt. Während im 1. Jahrzehnt (1990-1999) der Datenerfassung das Durchschnittsalter der Patienten mit schweren Hautreaktionen bei 48 Jahren lag, beträgt es im 2. Jahrzehnt 55 Jahre $[1,38]$. Die Letalität ist mit ca. $9 \%$ für SJS, ca. $29 \%$ für SJS/TEN-Übergangsform und ca. $48 \%$ für TEN sehr hoch [38]. Verglichen mit früheren Jahren scheint die Sterberate gestiegen, was wahrscheinlich auf das höhere Alter der Patienten - in einer älteren Gesamtpopulation und die damit verbundenen Grunderkrankungen zurückzuführen ist. Bei GBFDE ist die Prognose insgesamt besser, allerdings wurde bei älteren $\mathrm{Pa}$ tienten (über 70 Jahre) mit ausgedehnter Blasenbildung im Wiederholungsfall eine Sterberate von $20 \%$ beschrieben [7].

Mit Hilfe des o.g. SCORTEN lässt sich im Einzelfall eine prognostische Aussage treffen ( $\bullet$ Tab. 2). Im Verlauf von SJS und TEN kann es zu einer Begleithepatitis, einer tubulären Nephritis oder einer tracheobronchialen Mukosabeteiligung kommen, die sich in der Regel relativ rasch zurückbilden. Bei GBFDE wurden bislang keine systemischen Begleitreaktionen beschrieben.

Während die Hautveränderungen beim SJS und bei der TEN in der Regel narbenlos abheilen, bestehen als Folge der Entzündungsreaktion oft über Monate bis Jahre Hyper- und Hypopigmentierungen der Haut. Dies gilt auch für das GBFDE. Reversibles Effluvium sowie Nagelwachstumsstörungen bis hin zu einem vollständigen Nagelverlust wurden beobachtet. Problematischer sind Verwachsungen im Bereich der Schleimhäute, die zu Strikturen z.B. der Urethra oder des Ösophagus führen können. Die sicherlich gefährlichste und für den Patienten dramatischste Folgeerscheinung ist die Symblepharonbildung mit Entropium und Trichiasis, die zur Erblindung führen kann. Beim GBFDE wurden solche Komplikationen bislang nicht beobachtet. Hier besteht vielmehr ein großes Risiko, dass der Patient - nicht selten ein älterer Mensch im Pflegeheim mit rezidivierenden Harnwegsinfekten, der wiederholt z.B. Cotrimoxazol erhält - bei erneuter Einnahme des auslösenden Arzneimittels wieder ein, dann meist schwerer ausgeprägtes GBFDE erleidet.

Patienten, die eine schwere Arzneimittelreaktion der Haut überstanden haben, sollten einige Wochen nach der Entlassung aus dem Krankenhaus hautfachärztlich untersucht werden. Dabei können auch Folgeschäden festgestellt werden, die sich erst nach der Akutphase der Behandlung entwickelt haben, und entsprechende Maßnahmen eingeleitet werden.
Nach Entlassung aus der stationären Behandlung sollten Patienten dermatologisch weiter betreut und bei Vorliegen von Folgeschäden an entsprechende Fachkollegen überwiesen werden. Nicht nur die Akutbehandlung von schweren blasenbildenden Hautreaktionen sollte von Hautspezialisten koordiniert werden, sondern auch die Nachbetreuung.

\section{Zusammenfassung}

$\nabla$

Schwere bullöse Arzneimittelreaktionen wie Stevens-Johnson-Syndrom (SJS), toxisch epidermale Nekrolyse (TEN) und generalisiertes bullöses fixes Arzneiexanthem (GBFDE) sind spezifische Reaktionsformen, die aufgrund klinischer und histologischer Kriterien von anderen Dermatosen abgegrenzt werden können. Dabei werden SJS und TEN als ein Krankheitsbild von verschieden schwerer Ausprägung angesehen. Sie unterscheiden sich lediglich im Ausmaß der Hautablösung bezogen auf die Körperoberfläche. Wichtige Differenzialdiagnosen zu SJS/TEN sind das Erythema exsudativum multiforme majus (EEMM), welches aufgrund der typischen Hautveränderungen unterschieden werden kann und durch Infekte ausgelöst wird, sowie GBFDE. Während bei SJS/TEN mit Hilfe großer epidemiologischer Studien medikamentöse Risikofaktoren evaluiert werden konnten, liegen für GBFDE bislang nur Fallserien vor. In diesen fällt allerdings Cotrimoxazol als häufigster Auslöser vor allem bei rezidivierenden Fällen auf. Hinsichtlich der Behandlung schwerer Hautreaktionen stellt die Identifizierung des auslösenden Arzneimittels bereits den ersten Behandlungsschritt dar. Hinzu kommen in erster Linie supportive Maßnahmen, die auch eine adäquate Lokaltherapie einschließen. Immunmodulierende Substanzen wie Glukokortikosteroide, intravenöse Immunglobuline, Cyclosporin etc. können nach gründlicher Evaluierung der Gesamtsituation des betroffenen Patienten eingesetzt werden. Als Standardtherapie können sie allerdings nicht gelten. Zur Behandlung von Patienten mit schweren Hautreaktionen gehört auch das Vermeiden bzw. frühzeitige Erkennen von Folgeschäden, die sich vor allem im Bereich der Schleimhäute manifestieren. Da besonders die Augen langfristig Probleme machen können, empfiehlt sich die frühzeitige und enge Zusammenarbeit mit erfahrenen Augenärzten. Auch wenn Patienten mit TEN und ausgedehnter Hautablösung nicht immer in Hautkliniken und dermatologischen Abteilungen behandelt werden können, sondern intensivmedizinischer Betreuung bedürfen, sollten Dermatologen die Behandlung und auch die Nachbetreuung koordinieren.

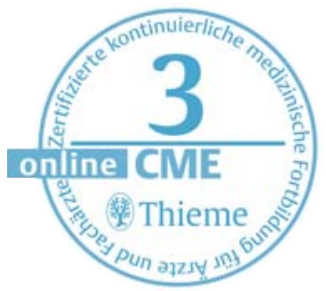


1 Mockenhaupt M, Norgauer J. Schwere arzneimittelinduzierte Hautreaktionen. Allergologie 2001; 9: 419-432

2 Stevens AM, Johnson FC. A new eruptive fever associated with stomatitis and ophthalmia: Report of two cases in children. Am J Dis Child 1922; 4: 526-533

3 Lyell A. Toxic epidermal necrolysis: an eruption resembling scalding of the skin. Br J Dermatol 1956; 68: 355 361

4 Hebra F von. Erythema exsudativum multiforme. In: Atlas der Hautkrankheiten, Kaiserliche Akademie der Wissenschaften. Wien, Austria: 1866: 54-57

5 Bastuji-Garin S, Rzany B, Stern RS et al. Clinical classification of cases of toxic epidermal necrolysis, Stevens-Johnson syndrome, and erythema multiforme. Arch Dermatol 1993; 129: $92-96$

6 Kauppinen K. Cutaneous reactions to drugs. With special reference to severe bullous mucocutaneous eruptions and sulphonamides. A clinical study. Acta Derm Venereol Suppl (Stockh) 1972; 68: 1-89

7 Mockenhaupt M, Herb T, Liss Y et al. Generalized bullous fixed drug eruption: analysis of 62 cases. 4th International Drug Hypersensivity Meeting, 22-25 April 2010, Rome, Italy. European Annals of Allergy and Clinical Immunology 2010; 42 (2): 43

8 Salopek TG. Nikolsky's sign: is it 'dry' or is it'wet'? $\mathrm{Br}$ 」 Dermatol 1997; 136: 762-767

9 Melish ME, Glasgow LA. The staphylococcal scalded skin syndrome: development of an experimental model. N Engl J Med 1970; 282: 1114-1119

10 Mockenhaupt M, Idzko M, Grosber M et al. Epidemiology of staphylococcal scalded skin syndrome in Germany. J Invest Dermatol 2005; 124: 700-703

11 Rzany B, Hering O, Mockenhaupt $M$ et al. Histopathological and epidemiological characteristics of patients with erythema exsudativum multiforme majus (EEMM), Stevens-Johnson syndrome (SJS) and toxic epidermal necrolysis (TEN). Br J Dermatol 1996; 135 (1): 6-11

12 Auquier-Dunant A, Mockenhaupt M, Naldi L et al. Correlations between clinical patterns and causes of erythema multiforme majus, Stevens-Johnson syndrome, and toxic epidermal necrolysis. Arch Dermatol 2002; 138: 1019 1024

13 Ziemer M, Wiesend CL, Vetter $R$ et al. Cutaneous adverse drug reactions to valdecoxib distinct from Stevens-Johnson syndrome and toxic epidermal necrolysis. Arch Dermatol 2007; 143: 711-716

14 Sidoroff A, Halevy S, Bouwes Bavinck JN et al. Acute generalized exanthematous pustulosis (AGEP) - A clinical reaction pattern. J Cutan Pathol 2001; 28: 113-119

15 Bocquet $H$, Bagot M, Roujeau JC. Drug-induced pseudolymphoma and drug hypersensitivity syndrome (Drug Rash with Eosinophilia and Systemic Symptoms: DRESS). Semin Cutan Med Surg 1996; 15: 250 - 257

16 Mockenhaupt M, Viboud C, Dunant A et al. Stevens-Johnson syndrome and toxic epidermal necrolysis: Assessment of medication risks with emphasis on recently marketed drugs. The EuroSCAR-study. I Invest Dermatol 2008; 128 (1): $35-44$

17 Halevy S, Ghislain PD, Mockenhaupt M et al. Allopurinol is the most common cause of Stevens-Johnson syndrome and toxic epidermal necrolysis in Europe and Israel. J Am Acad Dermatol 2008; 58 (1): 25-32

18 Arzneimittelkommision der Deutschen Ärzteschaft. Allopurinol ist die häufigste Ursache für Stevens-Johnson-Syndrom und Toxisch epidermaler Nekrolyse in Europa und Israel. Deutsches Ärzteblatt 2009; 36: 1753 -1754

19 Fagot JP, Mockenhaupt M, Bouwes Bavinck JN et al. Nevirapine and the risk of Stevens-Johnson syndrome or toxic epidermal necrolysis - preliminary results of a case-control study. AIDS 2001; $15: 1-6$
20 Dietrich A, Kawakubo Y, Rzany B et al. Low N-acetylating capacity in patients with Stevens-Johnson syndrome and toxic epidermal necrolysis. Exp Dermatol 1995; 4: 313-316

21 Nassif A, Moslehi H, Le Gouvello S et al. Evaluation of the potential role of cytokines in toxic epidermal necrolysis. J Invest Dermatol 2004; 123 (5): 850-855

22 Chung WH, Hung SI, Yang JY et al. Granulysin is a key mediator for disseminated keratinocyte death in StevensJohnson syndrome and toxic epidermal necrolysis. Nat Med 2008; 14 (12): $1343-1350$

23 Roujeau JC, Huynh TN, Bracq C et al. Genetic susceptibility to toxic epidermal necrolysis. Arch Dermatol 1987; 123: $1171-1173$

24 Chung WH, Hung SI, Hong HS et al. A marker for StevensJohnson syndrome. Nature 2004; 428: 486

25 Lonjou C, Thomas L, Borot $N$ et al. A marker for StevensJohnson-syndrome and toxic epidermal necrolysis: ethnicity matters. Pharmacogenomics Journal 2006; 6: 265 268

26 Hung SI, Chung WH, Liou LB et al. HLA-B*5801 allele as a genetic marker for severe cutaneous adverse reactions caused by allopurinol. Proc Natl Acad Sci USA 2005; 102: $4134-4139$

27 Lonjou C, Borot N, Sekula P et al. Stevens-Johnson syndrome and toxic epidermal necrolysis related to five high-risk drugs. Pharmacogenetics and Genomics 2008; 18 (2): $99-107$

28 Shiohara T, Mizukawa Y, Teraki Y. Pathophysiology of fixed drug eruption: the role of skin-resident $\mathrm{T}$ cells. Curr Opin Allergy Clin Immunol 2002; 2 (4): 317-323

29 Roujeau JC, Chosidow O, Saiag P et al. Toxic epidermal necrolysis (Lyell's syndrome). J Am Acad Dermatol 1991; 23: $1039-1058$

30 Struck MF, Hilbert $P$, Mockenhaupt $M$ et al. Severe cutaneous adverse reactions: emergency approach to nonburn epidermolytic syndromes. Intensive Care Med 2009; 36 (1): 22-32

31 Gueudry J, Roujeau JC, Binaghi M et al. Risk factors for the development of ocular complications of Stevens-Johnson syndrome and Toxic epidermal necrolysis. Arch Dermatol 2009; 145 (2): 157-162

32 Wolkenstein P, Latarje J, Roujeau J et al. Randomised comparison of thalidomide versus placebo in toxic epidermal necrolysis. Lancet 1998; 352: 1586-1589

33 Viard I, Wehrli P, Bullani R et al. Inhibition of toxic epidermal necrolysis by blockade of CD95 with human intravenous immunoglobulin. Science 1998; 282: 490-493

34 Faye 0 , Roujeau JC. Treatment of epidermal necrolysis with high-dose intravenous immunoglobulins (IVIG). Clinical experience to date. Drugs 2006; 65 (15): 2085 2090

35 Bachot N, Revuz J, Roujeau JC. Intravenous immunoglobulin treatment for Stevens-Johnson syndrome and toxic epidermal necrolysis. A prospective noncomparative study showing no benefit on mortality or progression. Arch Dermatol 2003; 139: 33-36

36 Bastuji-Garin S, Fouchard N, Bertocchi M et al. SCORTEN: a severity-of-illness score for toxic epidermal necrolysis. J Invest Dermatol 2000; 115: 149-153

37 Schneck J, Fagot JP, Sekula P et al. Effects of treatments on the mortality of Stevens-Johnson syndrome and toxic epidermal necrolysis: A retrospective study on patients included in the prospective EuroSCAR Study. J Am Acad Dermatol 2008; 58 (1): 33-40

38 Mockenhaupt M. Severe cutaneous adverse reactions. In: Burgdorf WHC, Plewig G, Wolff $\mathrm{HH}$, Landthaler M (Hrsg). Braun-Falco's Dermatology. 3. Aufl. Heidelberg: Springer, 2008: $473-484$ 


\section{CME-Fragen Schwere bullöse arzneimittel- induzierte Hautreaktionen}

1 Welche Aussage zu den schweren blasenbildenden Hautreaktionen trifft zu?

A Stevens-Johnson-Syndrom (SJS) und toxisch epidermale Nekrolyse (TEN) sind autoimmunologisch induzierte Reaktionsformen.

B SJS und TEN sind Hautreaktionen, die nach der Akutphase immer problemlos abheilen.

C Bei SJS und TEN sind spezifische Laborparameter im peripheren Blut diagnoseweisend.

D SJS und TEN können als eine Krankheitsentität mit verschiedenem Schweregrad aufgefasst werden.

E SJS und TEN unterscheiden sich histologisch grundlegend.

2 Welche Aussage(n) trifft/treffen zu? Das generalisierte bullöse fixe Arzneiexanthem (GBFDE)

1 ist eine multilokuläre, ausgedehnte Form des fixen Arzneiexanthems, welche mit Blasenbildung einhergeht.

2 unterscheidet sich histologisch eindeutig von SJS und TEN.

3 tritt häufig bei Patienten auf, die bereits früher ein fixes Arzneiexanthem hatten.

4 tritt mit hoher Wahrscheinlichkeit erneut auf, wenn das auslösende Arzneimittel wieder eingenommen wird.

5 hat eine deutlich bessere Prognose als die TEN.

A Nur die Antworten 1 und 2 sind richtig.

B Nur die Antworten 1, 3, 4 und 5 sind richtig.

C Nur die Antworten 2, und 4 sind richtig.

D Nur die Antworten 1, 2, 4 und 5 sind richtig.

E Alle Antworten sind richtig.

3 Wodurch unterscheiden sich Stevens-Johnson-Syndrom (SJS) und Erythema exsudativum multiforme majus (EEMM) nicht?

A Art der Einzeleffloreszenzen

B demografische Faktoren

C hämorrhagisch-erosive Schleimhautbeteiligung

D Verteilung der Läsionen

E Ätiologie

4 Welche Aussage ist richtig?

Zu den wichtigsten Differenzialdiagnosen von SJS und TEN zählt folgende Erkrankung, die sich in ihrer Histologie eindeutig unterscheidet:

A generalisiertes bullöses fixes Arzneimittelexanthem (GBFDE)

B bullöse Dermatitis solaris

C Verbrühung mit Blasenbildung

D Staphylococcal scalded Skin Syndrome (SSSS)

E Erythema exsudativum multiforme majus (EEMM)
5 Welche Aussage trifft für den SCORTEN Zu?

A Es handelt sich um ein System zur Abgrenzung der TEN von anderen Arzneimittelreaktionen.

B Der SCORTEN hilft bei der Kausalitätsbewertung von Arzneimitteln, die TEN auslösen können.

C Er spiegelt das zehnfache Risiko der TEN im Vergleich zu anderen Arzneimittelreaktionen wider.

D Er kann auch für die Einschätzung des Schweregrads von verschiedenen Arzneimittelreaktionen verwendet werden.

E Der SCORTEN erlaubt die Einschätzung der Prognose von Patienten mit SJS und TEN.

6 Welche Substanzen sind nicht als hochverdächtige Auslöser von Stevens-Johnson-Syndrom (SJS) und toxisch epidermale Nekrolyse (TEN) bekannt?

A Betablocker

B Allopurinol

C nicht steroidale Antirheumatika vom Oxicam-Typ

D antibakterielle Sulfonamide

E Lamotrigin

7 Welche Antwort zu SJS und TEN

ist richtig?

A Für die ätiologische Abklärung der TEN stehen spezifische In-vitro- und In-vivo-Testverfahren zur Verfügung.

B Das auslösende Arzneimittel wird oftmals einige Wochen lang gut vertragen, bevor es zum Auftreten von SJS und TEN kommt.

C Die Blasenbildung bei SJS und TEN tritt in der Regel unmittelbar nach der ersten Einnahme des auslösenden Arzneimittels auf.

D Das Auftreten von SJS und TEN setzt eine vorherige Sensibilisierung gegenüber einem Arzneimittel voraus.

E Die TEN tritt in der Regel rezidivierend auf.

8 Welche Aussagen treffen zu?

1 Bei wiederholtem Auftreten von GBFDE kann das Ausmaß der Hautablösung beträchtlich sein und eine TEN imitieren.

2 Pathogenetisch gibt es offenbar keinerlei Unterschiede zwischen SJS/TEN und GBFDE.

3 Das Auftreten von Blasen in den gleichen Lokalisationen wie beim Vorereignis scheint bei GBFDE durch ortsständige Memory-T-Zellen mediiert zu sein.

4 GBFDE wird häufig durch Cotrimoxazol ausgelöst, aber auch durch verschiedene Analgetika.

5 GBFDE kommt meist bei Kindern vor.

A Nur die Antworten 1 und 2 sind richtig.

B Nur die Antworten 2 und 3 sind richtig.

C Nur die Antworten 1, 3, und 4 sind richtig.

D Nur die Antworten 3, 4 und 5 sind richtig.

E Alle Antworten sind richtig. 
Welche Aussagen zur Pathogenese schwerer Hautreak-

tionen treffen zu?

1 SJS und TEN können nur auftreten, wenn zuvor eine entsprechende Sensibilisierung auf das verdächtige Arzneimittel stattgefunden hat.

2 Bei SJS/TEN handelt es sich um eine spezifische Reaktionsform, bei welcher zytotoxische T-Zellen und verschiedene Zytokine zu einem apoptotischen Prozess in der Epidermis führen.

3 In der Regel treten SJS und TEN im Rahmen der Erstanwendung eines Arzneimittels auf.

4 Eine genetische Prädisposition scheint bei SJS/TEN vorzuliegen, bei der der HLA-Locus auf Chromosom 6 eine Rolle spielt.

5 EEMM wird als IgE-vermittelte Reaktion aufgefasst.

A Nur die Antworten 1 und 2 sind richtig.

B Nur die Antworten 2, 3 und 4 sind richtig.

C Nur die Antworten 2, 3 und 5 sind richtig

D Nur die Antworten 1, 2 und 3 sind richtig

E Alle sind richtig.
10

Welche Aussagen zur Therapie von SJS und TEN treffen zu?

1 Ein interdisziplinäres Vorgehen ist unbedingt anzuraten, v. a. wenn große Areale der Haut und/oder die Schleimhäute massiv betroffen sind.

2 Die supportive Therapie spielt nur eine untergeordnete Rolle.

3 Eine frühzeitige und regelmäßige ophthalmologische Betreuung kann helfen, chronische und irreversible Folgeschäden an den Augen zu verhindern.

4 Intravenöse Immunglobuline sind immer das Mittel der Wahl, da sie sofort die Hautablösung zum Sistieren bringen und so den Tod der Patienten verhindern.

5 Eine desinfizierende Lokaltherapie ist nicht nötig, da die Hautoberfläche immer steril ist.

Nur 1 und 3 sind richtig.

Nur 2 und 3 sind richtig.

Nur 2, 3 und 5 sind richtig.

Nur 2, 4 und 5 sind richtig.

Alle sind richtig.

\section{CME-Fortbildung mit der Aktuellen Dermatologie}

Zertifizierte Fortbildung Hinter der Abkürzung CME verbirgt sich „continuing medical education“, also kontinuierliche medizinische Fort- und Weiterbildung. Zur Dokumentation der kontinuierlichen Fortbildung der Ärzte wurde das Fortbildungszertifikat der Ärztekammern etabliert. Hauptzielgruppe für das Fortbildungszertifikat sind Ärzte mit abgeschlossener Facharztausbildung, die im 5-jährigen Turnus einen Fortbildungsnachweis erbringen müssen. Es ist jedoch auch für Ärzte im Praktikum bzw. in der Facharztweiterbildung gedacht.

Die Fortbildungseinheit In den einheitlichen Bewertungskriterien der Bundesärztekammer ist festgelegt: „Die Grundeinheit der Fortbildungsaktivitäten ist der Fortbildungspunkt. Dieser entspricht in der Regel einer abgeschlossenen Fortbildungsstunde (45 Minuten)“. Für die erworbenen Fortbildungspunkte muss ein Nachweis erbracht werden. Hat man die erforderliche Anzahl von 250 Punkten gesammelt, kann man das Fortbildungszertifikat bei seiner Ärztekammer beantragen, welches man wiederum bei der KV (niedergelassene Ärzte) oder bei seinem Klinikträger (Klinikärzte) vorlegen muss.

Anerkennung der CME-Beiträge Die Fortbildung in der Aktuellen Dermatologie wurde von der Nordrheinischen Akademie für ärztliche Fort- und Weiterbildung für das Fortbildungszertifikat anerkannt, das heißt, die Vergabe der Punkte kann direkt durch die Thieme Verlagsgruppe erfolgen. Die Fortbildung in der Aktuellen Dermatologie gehört zur Kategorie „strukturierte interaktive Fortbildung“. Entsprechend einer Absprache der Ärztekammern werden die von der Nordrheinischen Akademie für ärztliche Fort- und Weiterbildung anerkannten Fortbildungsveranstaltungen auch von den anderen zertifizierenden Ärztekammern anerkannt.
Datenschutz Ihre Daten werden ausschließlich für die Bearbeitung dieser Fortbildungseinheit verwendet. Es erfolgt keine Speicherung der Ergebnisse über die für die Bearbeitung der Fortbildungseinheit notwendige Zeit hinaus. Die Daten werden nach Versand der Testate anonymisiert. Namens- und Adressangaben dienen nur dem Versand der Testate. Die Angaben zur Person dienen nur statistischen Zwecken und werden von den Adressangaben getrennt und anonymisiert verarbeitet.

Teilnahme Jede Ärztin und jeder Arzt soll das Fortbildungszertifikat erlangen können. Deshalb ist die Teilnahme am CME-Programm der Aktuellen Dermatologie nicht an ein Abonnement geknüpft! Die Teilnahme ist im Internet unter http://cme.thieme. de möglich. Man muss sich registrieren, wobei die Teilnahme an Fortbildungen abonnierter Zeitschriften ohne Zusatzkosten möglich ist.

Teilnahmebedingungen Für eine Fortbildungseinheit erhalten Sie 3 Fortbildungspunkte im Rahmen des Fortbildungszertifikates. Hierfür müssen $70 \%$ der Fragen richtig beantwortet sein.

CME-Fortbildung für Nicht-Abonnenten Teilnehmer, die nicht Abonnenten der Aktuellen Dermatologie sind, können für die Internet-Teilnahme dort direkt ein Guthaben einrichten, von dem pro Teilnahme ein Unkostenbeitrag abgebucht wird.

Teilnahme online unter http://cme.thieme.de 